\title{
Self-organzied Critical Control for the European XFEL using Black Box Parameter Identification for the Quench Detection System
}

\author{
Ayla S. Nawaz ${ }^{1}$, Sven Pfeiffer ${ }^{1}$, Gerwald Lichtenberg ${ }^{2}$, Holger Schlarb ${ }^{1}$
}

\begin{abstract}
The European Free Electron Laser (XFEL) consists of a large and complex plant, with many cost intensive and technological high-end components. It is therefore important that the XFEL can be operated reliably and safely using exception handling and fault detection systems. A crucial part of the system are the superconducting cavities for which especially quenches, i.e. the break down of the superconductivity have to be avoided. The paper shows the interaction of the fault detection system with the Low Level RF (LLRF) control system to maximize the field gradients. This is an example for process supervision, which can neither be classified as fault-tolerant, nor is it a reconfiguration system, but uses the result of the fault detection to operate the system at its fault critical limit. This scheme simulates a system which behaves like a selforganized critical system, and drives the process at its critical performance limit. It is therefore called Self-organized Critical Control (SOCC). The paper shows the basic set-up and quench detection methods of the European XFEL and gives an example for an application of SOCC.
\end{abstract}

\section{INTRODUCTION}

Fault tolerant control systems are developed to enhance the efficiency and performance of processes while ensuring a safer and more reliable system operation. The fault diagnosis result is used to adequately react to the respective faults in a process. Reconfiguration schemes use the diagnosis results and aim to keep the process running as closely as possible to its nominal operation, [1]. The reaction to a fault aims at reproducing the original operation of the process without risking any further damages or failures. In this paper an application is presented in which the operation performance of the system can be maximized by using the fault detection result, but without reconfiguring the behavior of the controller. The idea is, to operate the system close to its fault critical limit and by doing so, optimizing its performance. This is achieved by constantly provoking a known fault. As soon as a fault is detected, the operation point is adjusted such, that a failure of the system is avoided. For such an operation a reliable detection of incipient faults is necessary. Operating a system close to its limits can be compared to self organized critical systems in nature and since the proposed method simulates such a behavior, it is called Selforganized Critical Control (SOCC). The implementation of a SOCC is tested for one performance limit known to the European X-ray Free Electron Laser (XFEL). The European XFEL is a research project currently under construction at the Deutsches Elektronen Synchrotron (DESY). It will

\footnotetext{
${ }^{1}$ Deutsches Elektronen Synchrotron, Hamburg, Germany $\{a y l a . n a w a z$, sven.pfeiffer, holger.schlarb\}@desy.de University of Applied Sciences Hamburg, Germany gerwald.lichtenberg@haw-hamburg. de
}

supply pulsed laser light with a tunable wavelength in the X-ray range $\left(10^{-10} \mathrm{~m}\right)$ and a repetition rate of maximum $4.5 \mathrm{MHz},[2]$. To reach this short laser light wavelength, the European XFEL is operated in pulsed mode with a $10 \mathrm{~Hz}$ repetition rate. Electrons will have to be accelerated to a maximum target energy of $17.5 \mathrm{GeV}$. This is achieved by increasing the energy of free electrons through an interaction with electromagnetic radio frequency (RF) waves inside so called cavities. these cavities are superconducting RF cavities built from pure Niobium and are high quality resonators, which are operated at a frequency of $1.3 \mathrm{GHz}$ and field gradients up to $30 \mathrm{MV} / \mathrm{m}$.

In order to provide high brightness and narrow bandwidth laser light in the X-ray range, it is required to control the electromagnetic fields inside these cavities with extremely high accuracy of $0.01 \%$ root mean square (rms) in amplitude and $0.01 \mathrm{deg}$ (rms) in phase. The control system for the cavities is embedded into the Low Level Radio Frequency (LLRF) system. Using multiple sensors to obtain information about the state of the cavity closes the RF field feedback loop. This not only includes the measured input signals to obtain the electromagnetic field and the output signals measuring the obtained electromagnetic fields, but also different temperature and pressure measurements.

Due to the size of the accelerator and the relatively large number of components, a high degree of automation is expected to assist operators. This includes the detection and handling of faults. After the XFEL is finally assembled, the question of performance arises. Some of the performance limits are well known. The maximum gradient of $30 \mathrm{MeV}$, for example is often not reached due to a performance limiting fault called quench. In this paper the detection of quenches, is used to exemplify an application for a Self-organized Critical Control.

In Section II, the cavity system of the XFEL is introduced, to give some insight into the complexity of the supervision problem. Section III then introduces the system identification based fault detection system used to detect the quenches. It discusses the residual generation, the residual evaluation and model uncertainties. In Section IV the idea of Self-organized Critical Control is introduced and Section V gives some first attempts to implement it for the XFEL control loop with real data test results. Finally, the weaknesses of the developed fault tolerant control system are put together in the summary.

\section{Cavity System of the European XFEL}

Each cavity is used as an electromagnetic field resonator. The quality of the resonator is determined by how little 
energy is lost through the cavity walls, when it resonates. For the XFEL Niobium is used as the superconducting material for the cavities, which obtains very high quality factors. The superconductivity of the cavities can only be guaranteed when they are operated at a temperature below $4 \mathrm{~K}$, and they are therefore normally operated at $2 \mathrm{~K}$, [8]. The cooling is done with cryogenic modules, in which helium is used as a cooling material. Each cryogenic module hosts eight cavities being cooled down simultaneously. Due to economic reasons, up to four cryogenic modules are grouped for one RF station, resulting in a total of $25 \mathrm{RF}$ stations for the entire XFEL. They are operated by the LLRF controller, where a maximum of 32 cavities are controlled using the vector sum of the individual cavity signals. An overview of such an RF station is depicted in Fig. 7.

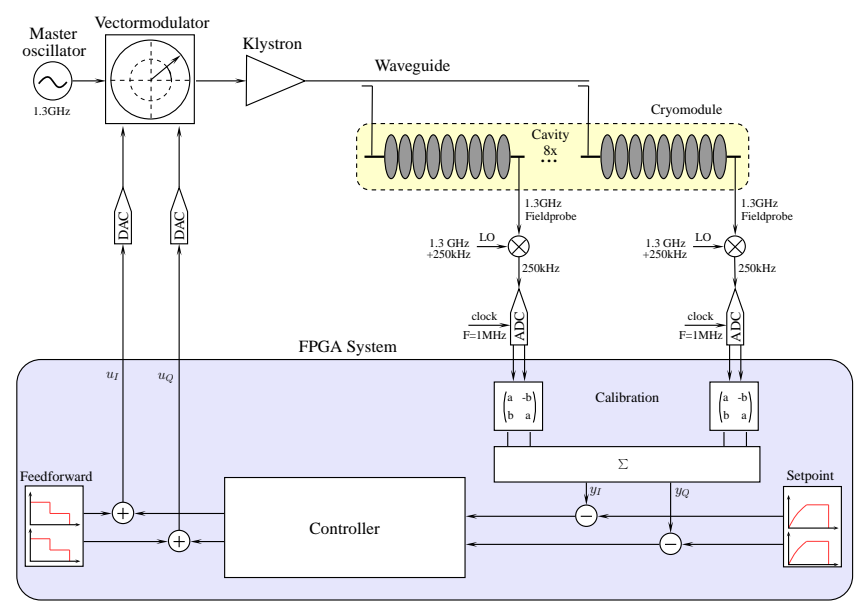

Fig. 1: Overview over the LLRF control scheme for the European XFEL

\section{A. LLRF system description}

The master oscillator (MO) provides a constant RF signal at $1.3 \mathrm{GHz}$ to each RF station. This MO signal can be changed by a vector modulator in amplitude and phase, before it is amplified by the klystron and distributed through a wave guide system to the RF cavities. For each cavity the forward and the reflected power signals are measured by pick-up antennas, as is the power of the standing waves inside the cavity, the probe signal. The signals are downconverted from $1.3 \mathrm{GHz}$ to an intermediate frequency of $54 \mathrm{MHz}$, and further sampled by an analog to digital converter (ADC) with a sampling frequency of $9 \mathrm{MHz}$. The measured probe signals from the individual cavities are summed up, and the vector sum is processed by the control hardware. Computing the error of vector sum and set-point and from this the optimal drive signal closes the control loop. The cavities are operated in pulsed mode, hence the forward signal is periodically ramped-up and shut-down, resulting in the desired pulse shape for the probe signal, see Fig2.

\section{B. Detuning}

The frequency of the MO signals is ideally also the resonance frequency of each cavity. Any deviation of the

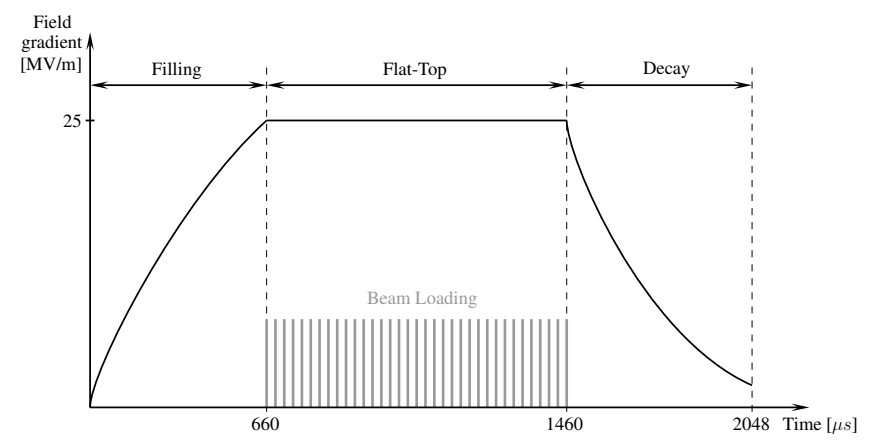

Fig. 2: The repetition rate of these pulses is $10 \mathrm{~Hz}$, any reaction to a detected fault has to be faster than $100 \mathrm{~ms}$.

resonance frequency leads to a loss of cavity gradient. The cavity detuning is in the order of a few hundreds Hertz. For the standard operation of the European XFEL the rough detuning of each cavity is minimized by changing the dimensions of the resonator with the help of stepper motors, while the fine tuning, which counteracts any detuning that happens during one pulse, is performed using piezo electric actuators.

\section{Quench Detection USING ONLINE System IDENTIFICATION}

In order to understand why a system identification approach was used, it is necessary to introduce the cavity model and some characteristics of a quench. The following will also explain, why it is desirable to operate the cavities at their quench limit and use the proposed SOCC.

\section{A. Quench Description}

Obtaining a pure Niobium cavity is not trivial. Small impurities in the material can never be avoided. These impurities cause energy loss. The higher the field gradient in the cavity is, the higher are the losses at these impurities. When the losses become so high, that they start heating the surrounding material, and thereby reduce the superconducting to normal conducting behavior, the cavity quenches. The maximally possible field gradient before a cavity quenches is different for each cavity and therefore this maximum gradient limit has to be tested before assembling each cavity into the XFEL. The cavities are then operated such, that a desirable maximum amplitude of the probe signal during the flattop is reached, while staying below the critical limit above which the weakest cavity of the LLRF system quenches. The break down of the gradient field in one individual cavity can happen in just a few consecutive pulses, or in just one single pulse. This is depicted in Fig. 3, where the black dashed line shows nominal behavior followed by three consecutive pulses leading to a complete breakdown of the gradient field. In order to detect quenches, the current quench detection system makes use of the loaded quality factor $Q_{L}$. Unfortunately, the $Q_{L}$ value is also dependent on the external quality factor $Q_{\text {ext }}$, which gives information about the amount of coupling of the electromagnetic field into the cavity. The dependency 

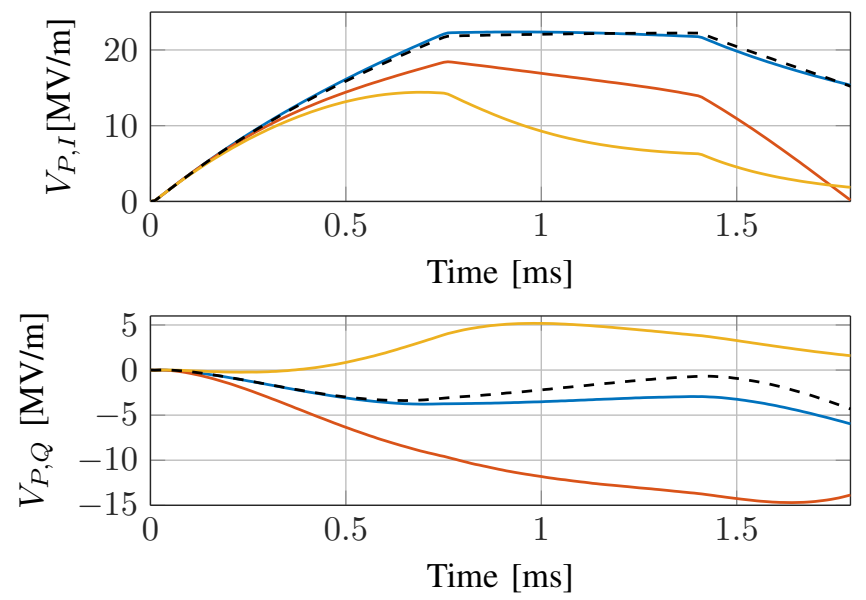

Fig. 3: Cavity probe signal for four RF pulses. Nominal (-- -), and three consecutive pulses with quench behavior $(-,-,-$

is described by:

$$
\frac{1}{Q_{L}}=\frac{1}{Q_{e x t}}+\frac{1}{Q_{0}}
$$

A typical $Q_{\text {ext }}$ value is $Q_{\text {ext }}=3 \cdot 10^{6}$ and a typical drop of the $Q_{0}$ values during a quench is from $2 \cdot 10^{10}$ to about $10^{7}-10^{8}$. This means that a two-order of magnitude drop in $Q_{0}$ will only result in $<2 \%$ decrease in measurable $Q_{L}$, while the dissipated heat induced by a quench scales as $1 /$ $Q_{0}$. Possible fluctuations of $Q_{0}$ can therefore not easily be seen in the $Q_{L}$ values, [3]. Using the $Q_{L}$ values to detect quenches can only be done, when the external load $Q_{e x t}$ is kept constant, thus ensuring that any detected change in $Q_{L}$ really results from a change of the $Q_{0}$ value. This method is therefore not sufficiently accurate and makes the early detection of quenches, necessary for the proposed SOCC, difficult. However using the $\omega_{1,2}$ values as an indication for a quench is a reason to use system identification methods for the following fault detection system.

\section{B. Cavity Model}

The behavior of the cavity system is described by a second order grey box model. The in- and output signals for the cavity are represented by the complex forward and probe signals, $\mathbf{V}_{F}=V_{F, I}+j V_{F, Q}$ and $\mathbf{V}_{P}=V_{P, I}+j V_{P, Q}$, respectively. For the grey box model, the in-phase and quadrature components are considered as two separate inputs. The model is thus represented by a multiple-input multiple-output (MIMO) state space model, [4]. It is given by

$$
\begin{aligned}
\frac{d}{d t}\left[\begin{array}{c}
V_{P, I}(t) \\
V_{P, Q}(t)
\end{array}\right] & =\overbrace{\left[\begin{array}{cc}
-\omega_{1 / 2} & -\Delta \omega \\
\Delta \omega & -\omega_{1 / 2}
\end{array}\right]}^{A}\left[\begin{array}{c}
V_{P, I}(t) \\
V_{P, Q}(t)
\end{array}\right] \\
& +\overbrace{\left[\begin{array}{cc}
\omega_{1 / 2} & 0 \\
0 & \omega_{1 / 2}
\end{array}\right]}^{B}\left[\begin{array}{c}
V_{F, I}(t) \\
V_{F, Q}(t)
\end{array}\right], \\
{\left[\begin{array}{c}
V_{P, I}(t) \\
V_{P, Q}(t)
\end{array}\right] } & =\underbrace{\left[\begin{array}{ll}
1 & 0 \\
0 & 1
\end{array}\right]}_{C}\left[\begin{array}{c}
V_{P, I}(t) \\
V_{P, Q}(t)
\end{array}\right],
\end{aligned}
$$

with system matrix $A$, input matrix $B$, output matrix $C$ and input and output vector given by the forward and probe signals respectively, [5]. The cavity quantities, bandwidth $\omega_{1 / 2}$ and detuning $\Delta \omega$, are reflected in the system $A$ matrix, as well as in the diagonal of the B matrix of the grey box model. The dynamic behavior is described by the eigenvalues of the state space model which are given by $\lambda_{1 / 2}=-\omega_{1 / 2} \pm j \Delta \omega$. Knowing that a quench leads to a change of $\omega_{1 / 2}$ and $\Delta \omega$, the identification of the eigenvalues of the system A matrix as described by the grey box model cavity system are used for the quench detection system.

\section{Residual Generation using System Identification}

The idea of the system identification based approach quench detection system is to identify the eigenvalues of the above described MIMO system for each RF pulse. This means, that the measured in-phase, as well as quadrature cavity signals obtained from one pulse, are used for the system identification. The nominal eigenvalues are set to the mean of 50 consecutive non quenching and nominal pulses. The eigenvalues of the system are a complex conjugate pole pair and the value of the real and imaginary part are used as two individual values in the residual generation. The residual vector is described by:

$$
\left[\begin{array}{l}
r_{1} \\
r_{2}
\end{array}\right]=\left[\begin{array}{c}
\omega_{n 1 / 2}-\omega_{1 / 2} \\
\Delta \omega_{n}-\Delta \omega
\end{array}\right]
$$

with $\mathrm{n}$ denoting that the parameter value is obtained from nominal data sets and $r_{1}, r_{2}$ representing the residuals. The system identification itself requires a prediction based method to identify the described grey box model. This method requires to solve an optimization problem. Therefore, instead of using a grey box model structure, a black box system identification is chosen, which uses subspace methods that is more time efficient than the prediction based methods. Instead of identifying the values of $\omega_{1 / 2}$ described by the grey box model, the eigenvalues of the cavity systems black box model are identified. Again, using the real and imaginary components of the identified eigenvalues of system matrix A as two components of the residual, shows a sensitivity towards quenches. The used residual is therefore described by the two dimensional vector:

$$
\left[\begin{array}{l}
r_{1}(p) \\
r_{2}(p)
\end{array}\right]=\left[\begin{array}{l}
\operatorname{Re}\left\{\lambda_{1 n}\right\}-\operatorname{Re}\left\{\lambda_{1}\right\}(p) \\
\operatorname{Im}\left\{\lambda_{1 n}\right\}-\operatorname{Im}\left\{\lambda_{1}\right\}(p)
\end{array}\right]
$$


Where $\mathrm{p}$ reflects that the residual is obtained for each pulse. In order to understand the results obtained by this system identification approach it has to be mentioned, that the subspace method chosen here, not only identifies the parameters of the $A, B$ and $C$ matrices, but also considers and identifies a disturbance matrix $K$.

$$
\begin{aligned}
\frac{d}{d t} V_{P}(t) & =A V_{P}(t)+B V_{F}(t) \\
V_{P}(t) & =C V_{P}(t)+K E_{F}(t)
\end{aligned}
$$

where $E(t)=\left[e_{F, I}(t), e_{F, Q}(t)\right]^{T}$ denotes the disturbance, [13].

\section{Available Data}

The amount of available data showing quenches is limited. Therefore, the development of the quench detection system had to as a first step, rely on artificial quenches, see Fig. 4. These are obtained by the manipulation of nominal data sets, which roughly depict the behavior known from real quench data sets. In the second step, real quench data is used.
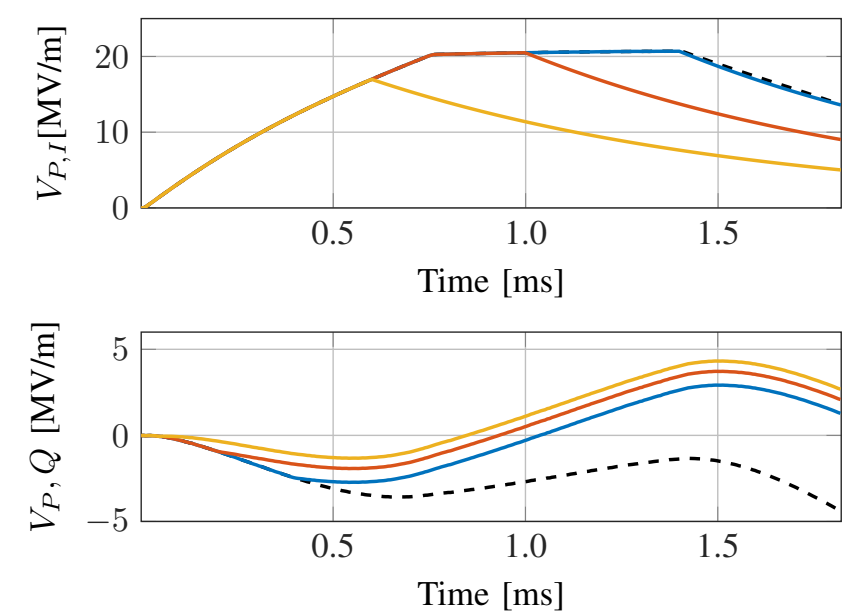

Fig. 4: Three artificial quench stages: Stage $1(-)$, Stage $2(-)$, Stage $3(-)$, and the nominal behavior $(---)$ of the signal.

\section{E. Residual Evaluation}

In order to define a boundary between acceptable residual values and values that depict a quench, the residuals obtained from the artificial quench data sets are depicted in Fig. 5. The boundary is chosen by using a linear Support Vector Machine (SVM), a tool known to machine learning, [9]. To train the Support Vector Machine, a set of parameter values is necessary, which depict nominal and quench cavity behavior. The boundary obtained through this procedure ensures a maximal margin to those residual values, which are closest together and belong to the two different classes (nominal/quench) on the 2-dimensional plane. However, the boundary obtained for the artificial quenches proved to be inadequate when using real data measurements. In practice, all so far observed quenches can be caught when either $\operatorname{Re}\left\{\lambda_{1}\right\}$ exceeds a certain value, or $\operatorname{Im}\left\{\lambda_{1}\right\} \neq 0$. This poses

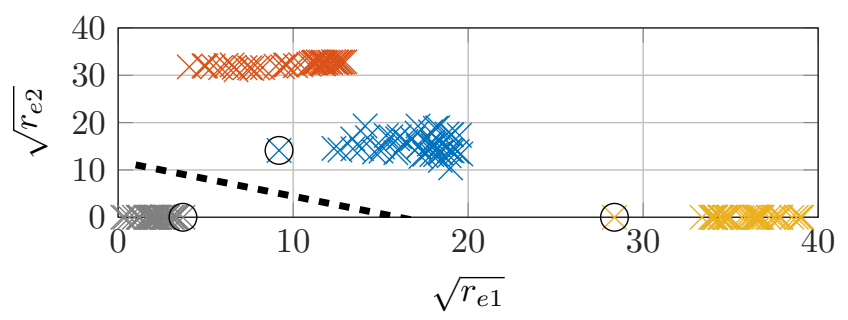

Fig. 5: Each data point depicts the residual value of one system identifiaction, using artificial quenches. The dashed line is the boundary obtained from the Support Vector Algorythm (-- ). Stage1: $\times$ Stage2: $\times$ Stage3: $\times$, Nominal: $\chi$.

a hard boundary on the $\operatorname{Im}\left\{\lambda_{1}\right\} \neq 0$. The $\operatorname{Re}\left\{\lambda_{1}\right\}$ values are considered as faulty, when the residual exceeds two times the standard deviation of the nominal $\operatorname{Re}\left\{\lambda_{1}\right\}$ values. Fig. 6 shows how the new quench detection system, using the described residuals, is more sensitive to a quench, than the current quench detection system. The red point indicates the first pulse which shows incipient quench behavior.
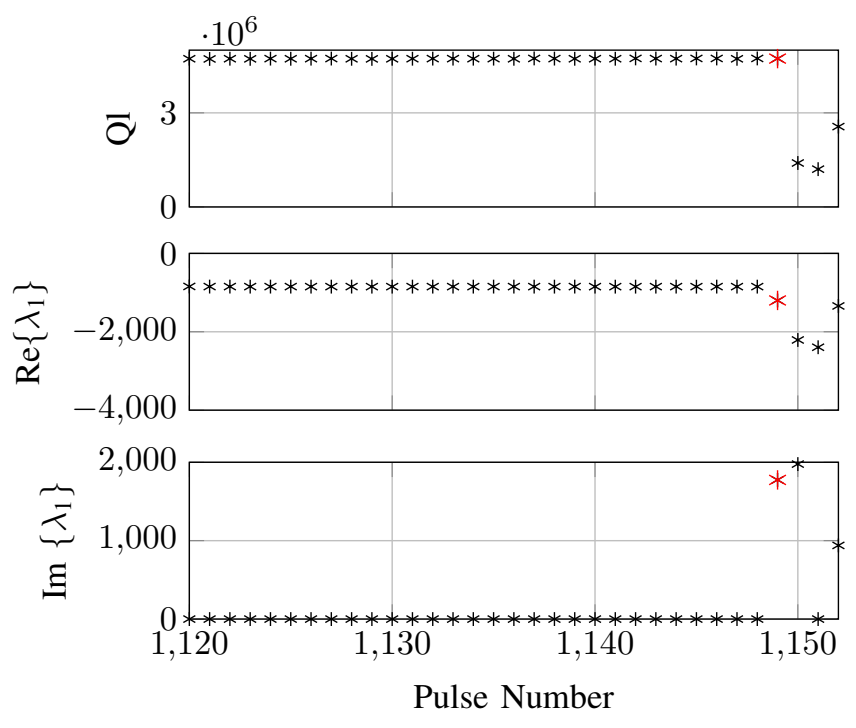

Fig. 6: The red value indicates the first pulse showing incipient fault behavior. Where the current quench detection value is not separable from nominal behavior, the model based detection shows a detectable change of value

\section{F. Model Uncertainties of Identified Parameters}

The fit of the output of the system model to the output obtained by the real system, using the same input signals, gives an indication about model uncertainties. The fit is commonly given in $\%$, i.e. $100 \%$ when the measured signal and the signal obtained by the model are identical. The output of the system model can, however, be obtained either by considering the system model including the disturbance matrix $K$, which is known as the fit of prediction, or by neglecting the disturbance matrix $K$, which is then called the fit to simulation. In nominal operation the values of $r_{1}(p)=$ $0 \pm \sqrt{\sigma}$, with $\sigma$ denoting the standard deviation change 
considerably, $\Delta r_{1}(p)>2 \sqrt{\sigma}$, when the system output shows non nominal behavior. The nominal behavior of $r_{2}(p)=0$, and only change, when the output signals have diverged considerably from the nominal values. The parameters used to obtain these residuals are estimated with a fit to prediction of $99 \%$ and $93 \%$ for the real and imaginary components respectively. The fit to simulation values, however, show that the $V_{P, I}$ signal is $96 \%$ and $34 \%$ for the $V_{P, Q}$ signals. The parameter values, and therefore the residual values, more dominantly give information give information about the inphase system output. The quadrature output of the system is not well identified. For the quench detection system this fact has to be kept in mind, however, the in-phase signal is more important for the description of the system behavior.

\section{G. False Alarms and Undetected Faults}

a) False Alarms: Using in total 5000 nominally operating signals a False Alarm rate of $F A=0$ was obtained. It has to be noted, that the used signals were taken from closed loop operation, and the detuning of the cavity was held close to zero. This is also how the XFEL will be operated. However, it could be observed, that if the detuning is not set to zero, or changes over time, this is also reflected in the identified parameters of the system and therefore in the residual values. Therefore, a zero false alarm rate can only be obtained, when the detuning of the cavity is kept at a minimum.

b) Undetected Faults: Out of all available quench event measurements (15), all quenches were detected. Comparing the sensitivity towards faults to the current quench detection system it can be noted, that there are cases in which the system identification approach is more sensitive to a change of the output signals. On the other hand, no cases were observed in which the system identification system is less sensitive.

\section{Self-organized Critical Control (SOCC)}

Self Organised Criticality (SOC) is a property which describes complex physical systems and it has been used to model multiple system behaviors from different fields of research, [7]. The basic phenomena that are explained by SOC, can best be described by the sand pile example, [11]. When a sand pile is built by adding one grain at a time, the sand pile grows to a certain critical point. After that point, repeated avalanches reduce the size of the sand pile to the critical, self organised pile size. Using the notion of a system which is constantly held at its critical point, the Self Organised Critical Control scheme aims to operate a system at a state in which it is constantly prone to failure. This approach should not be confused with Self Organised Criticality Control, in which it is the aim to control a self organising system, [12].

The failure of the system is, however, avoided by reacting to the detection of a fault. This reaction will then set the operating point in such a way, that the detected fault can not lead to a failure. This process is then repeated, again setting the operation point to the state in which the risk of system failure is high. The following assumptions on the nominal

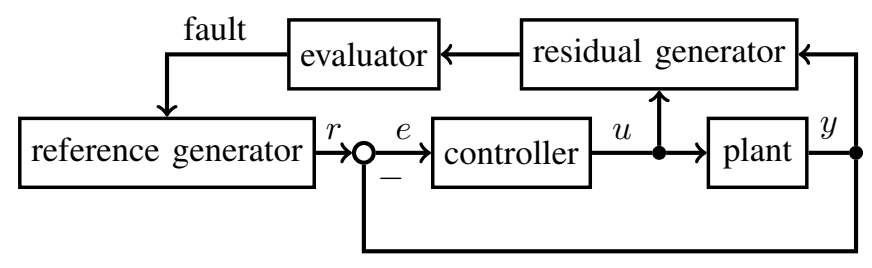

Fig. 7: Self-organized Critical Control Loop (SOOC)

and faulty behavior of the system are required to implement an SOCC as presented here:

1) occurrence of one fault depends on the operation point,

2) this fault can be detected in real time,

3) actions to increase fault risk by changing the set point usually to higher system performance - are applicable.

4) nominal behavior can be regained - after the fault is detected - by immediate setpoint changes.

The last point requires, that either an incipient fault can always be reliably detected, or the system reaction to a fault is faster than the failure of the component, when a fault occurs.

\section{SOCC FOR THE LLRF SYSTEM OF XFEL}

In the case of the described quenches the assumptions given in Section IV are met. Before the total break down due to a quench, the cavity mostly becomes only partially normal conduction (soft quenching). When this happens, a reduction of the cavity gradient set point can reduce the amount of energy lost through the cavity walls, giving the cryogenic system enough time to reduce the temperature of the cavity walls and resume being superconducting.

The cavity system is operated in closed loop. The signals of all cavities belonging to one RF station are used as the input to the controller. Any discrepancies from the specified flattop behavior in amplitude or phase are corrected. When a cavity quenches, the gradient in the cavity drops. In order to compensate the gradient loss of the module, the LLRF controller would ramp up the set-point of the vector sum gradient. If the quench is however detected, instead of compensating the gradient drop, the cavity is strongly detuned and taken out of the vector sum. Sometimes the cavity shows soft quenches for a couple of pulses before it quenches. If these kind of quenches can be detected reliably and quickly, a less drastic reconfiguration of the controller than done at the moment could be considered. In the case of a soft quench, the affected cavity would not be detuned from the rest of the RF system, but rather the set-point could be adjusted such, that the cryogenic module can recover itself and the loss of the quenching cavity can be avoided. Using the quench detection system to operate the LLRF system with an SOCC has two practical applications for the XFEL. The approach of operating a cavity at its quench limit can be used to periodically investigate the quench limit of the individual cavities. This information can add to the understanding of cavity wear and help to predict its long term behavior. Additionally a SOCC could be used to drive the entire XFEL 
to its performance limit. This approach maximizes the energy available for the acceleration of the electrons without risking any quenches. First tests only using one cavity can be seen in Fig. 8. The SOCC approach could also be used to operate the entire machine safely at its higher performance limits. The limiter value, limiting the field gradient of the cavity to below its previously determined quench limit is used as a first reference point, where quenches can be expected. The algorithm can be described as follows:

1) If field gradient $<$ quench limit, increase field gradient, steps of increase inversely proportional to distance to quench limit

2) If quench is detected, field gradient is set to the two step previous lower set point

3) If after a quench no quench is detected, field gradient is increased with a smaller step-size

4) If three quenches in a row are detected, the gradient is decreased considerably, giving the cavity time to recover

5) Reset and increase field gradient
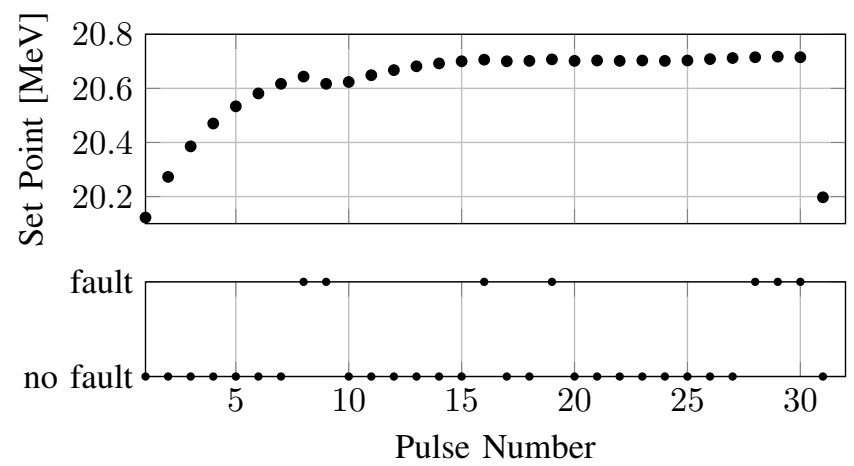

Fig. 8: Set-point value with the respective detection result. Set-point change is given as proposed for SOCC. Lowering the set-point when a quench is detected, increasing set- point when no quench is detected. Note, that between the pulses of 27 to 29 the setpoint seems not to be decreased, although a quench occurs. This is due to the fact, that the first two quenches are handled by setting the set-point value to the last non-quenching setpoint. It can be said, that the setpoint values have reached a saturation.

\section{SUMMARY}

For the European XFEL it is of interest to safely be able to obtain the highest energies possible for the accelerated electrons, without risking any damages to the machine. This is, however, limited due to various factors one of which is the possibility of quenches, when the field gradients are set too high in the superconducting cavities. It is therefore desirable have a controller scheme with which it is possible to drive each cavity into its quenching limit without really risking a severe damage. In this paper, a model based fault diagnosis procedure was presented which is able to detect incipient quenches. With the help of this detection system the idea of a Self-organized Critical Control (SOCC) was introduced. First on-line results of this procedure were taken at the XFEL test facility. These tests have shown, that the cavity can be operated near its quench limit, without risking any severe damages to the cavities. The detection procedure presented here uses the system identification method to obtain information about the systems' eigenvalues and generate a residual, which is sufficiently sensitive to the quench. This identification technique proved to be just as, or in some cases even more, reliable and sensitive towards quenches than the currently used fault detection scheme. It is therefore suitable to be used for the proposed SOCC, since it will protect the machine well before it really quenches. One major drawback of this procedure is, that the system identification results are not obtained fast enough, to be relevant for a fault detection system during nominal operation. Nevertheless, the SOCC can be used to periodically test the quench limits (using a smaller repetition rate of pulses) when the machine is checked during maintenance procedures. Furthermore, it is desirable to obtain residual values, which are not dependent on the detuning of the cavity, or the setpoint of the system. To do this, further investigation is necessary.

\section{REFERENCES}

[1] X. Qi, D. Theilliol, J. Qi, Y. Zhang, J. Han, D. Song, L. Wang, and Y. Xia, Fault diagnosis and fault tolerant control methods for manned and unmanned helicopters: A literature review, Conf. Control FaultTolerant Syst. SysTol, pp. 132139, 2013.

[2] V. Ayvazyan and S. N. Simrock, "EU contract number RII3-CT-2003506395 CARE Conf-04-027-SRF," 2003.

[3] V. Ayvazyan, O. Hensler, C. Schmidt, S. H., and J. Branlard, "Superconducting Cavity Quench Detection and Prevention for the European XFEL," in ICALEPCS2013: Proceedings of the 14th International Conference on Accelerator \& Large Experimental Physics Control Systems, 2013, p. 1239.

[4] S. Pfeiffer, "Symmetric grey box identification and distributed beambased controller design for free-electron lasers," Hamburg University of Technology, 2014.

[5] T. Schilcher, Vector Sum Control of Pulsed Accelerating Fields in Lorentz Force Detuned Superconducting Cavities, Thesis, 1998.

[6] Noura, Hassan and Didier, Theilliol and Jean-Chrisophe, Ponsart and Chamseddine, Abbas "Fault- tolerant Control Systems : Design and Practical Applications," 2009

[7] R. Frigg, "Self-organised criticality - What it is and what it isn't," Stud. Hist. Philos. Sci. Part C Stud. Hist. Philos. Biol. Biomed. Sci., vol. 34, no. 3, pp. 613632, 2003.

[8] M. Altarelli and Others, The European X-Ray Free-Electron Laser, no. July. 2007.

[9] L. Zhou, L. Wang, L. Liu, P. Ogunbona, and D. Shen, Support Vector Machines Applications, 2014.

[10] K. Kasprzak, D. Konwisorz, K. Krzysik, S. Myalski, J. Swierblewski, K. Turaj, M. Wiencek, A. Zwozniak, I. F. J. Pan, D. Kostin, and K. Przygoda, Automated Quench Limit Test Procedure for Serial Production of XFEL RF Cavities, pp. 78.

[11] A. Banerjee, Self-organized criticality in sandpile models, pp. 113, 2012.

[12] P. A. Noel, C. D. Brummitt, and R. M. DSouza, Controlling selforganizing dynamics on networks using models that self-organize, Phys. Rev. Lett., vol. 111, no. 7, pp. 15, 2013.

[13] L. Ljung, Aspects and Experiences of User Choices in Subspace Identification, 2003.

[14] S. Simani, C. Fantuzzi, and R. J. Patton, Model-based Fault Diagnosis in Dynamic Systems Using Identification Techniques. London: Springer London, 2003. 